\title{
Labyrinthe
}

4 | 1999

Numéro 4

\section{Maupassant et la vision fantastique}

\section{Laurent Dubreuil}

\section{OpenEdition}

\section{Journals}

Édition électronique

URL : http://journals.openedition.org/labyrinthe/294

DOI : $10.4000 /$ labyrinthe.294

ISSN : 1950-6031

Éditeur

Hermann

Édition imprimée

Date de publication : 1 octobre 1999

Pagination : 87-100

Référence électronique

Laurent Dubreuil, «Maupassant et la vision fantastique », Labyrinthe [En ligne], 4 | 1999, mis en ligne le 19 février 2005, consulté le 30 avril 2019. URL : http://journals.openedition.org/labyrinthe/294 ; DOI : $10.4000 /$ labyrinthe.294

Ce document a été généré automatiquement le 30 avril 2019

Propriété intellectuelle 


\title{
Maupassant et la vision fantastique
}

\author{
Laurent Dubreuil
}

1 Tout effort de définition du fantastique repose sur le dogme d'une reconnaissance possible. Question traversante : «à quoi voit-on qu'un texte est fantastique ? ». Il n'y a pas d'innocence critique et l'on ferait bien de toujours commencer par s'interroger sur les conditions de sa propre vision. La remarque vaut pour chaque domaine de la poétique générique ; elle s'impose de façon pressante dès qu'on traite du fantastique - qui naît du phantaneïsthaï (et pas du surnaturel ou des traces du folklore). Le fantastique est d'abord une affaire de vision, il s'élabore au plus près du processus de l'apparaître, il a trait à la sortie et l'entrée dans l'ombre, il suit ce visible qui à la fois se tapit dans sa cache d'invisibilité et va se rendre au plus haut point visible. Il remet en cause toute univocité régulière, il rend caduque la rationalité positive de la vue. Le fantastique transforme la perception.

2 Alors, plutôt que de s'intéresser aux évidences des prétendues « lois du genre », on doit étudier le fantastique à travers ce qu'il propose : la thématisation et le réseau philosophique du voir, de l'apparition, de l'invisible. Cette perspective peut ainsi projeter le fantastique sur le champ de la vision. Du coup, l'image ne se contente plus de deux dimensions ; elle prend le relief que laissait pour compte l'amblyopie de la narratologie.

Le fantastique - donc - est une histoire d'œil. Tout comme les récits de Maupassant auxquels le présent article se consacrera. En particulier, Le Horla, dans sa version de 1887, où se ressent la présence d'un autre. Une présence étrange, une présence invisible. Et d'invisible, il est incessamment question dans le journal intime du personnage principal.

Dès la date du douze mai (le récit commence le huit), le narrateur du Horla parle du " mystère de l'Invisible » (II, 914)2. L'Invisible porte sa capitale comme une marque de puissance. L'Invisible est presque dans le monde, il lui est plutôt coextensif, il forme un domaine pour tout ce qui est surnaturel. L'Invisible est une zone habitée. De là viennent sans doute les « êtres invisibles rôdant autour de l'homme ou rêvés par lui » (II, 931) dont parle « le grand traité du docteur Hermann Herestauss » (II, 930). 
5 Aussi est-il naturel de croire d'abord que le Horla, à la « présence invisible et constante » (II, 929), est lui-même un « invisible » (II, 930). Mais après la lecture approfondie du livre de Herestauss, le Je en vient à conclure que le Horla est un principe synthétique de l'Invisible. Son essence est élargie aux dimensions de tout le monde invisible ; et, le dix septembre, le dernier jour d'écriture, il est définitivement question de « l'Être Invisible et Redoutable » (II, 938).

6 Or, le texte le répète assez, l'invisible est inconnaissable, et réciproquement :

l'air invisible est plein d'inconnaissables Puissances (II, 914 ');

S'il existait sur la terre d'autres êtres que nous, comment ne les connaîtrions-nous point depuis longtemps ; et comment ne les auriez-vous pas vus, vous ? comment ne les aurais-je pas vus, moi ? (question posée par le narrateur à un moine sur le Mont Saint-Michel ; II, 918) ;

un être étranger, inconnaissable et invisible. (II, 920) ;

cet invisible ? cet inconnaissable. (II, 930) ;

ce corps transparent, ce corps inconnaissable. (II, 938)

7 D'un bout à l'autre du récit, la paronomase philosophique voir/savoir paraît être tenue. A priori, nulle connaissance délivrable sur ce qui échappe à la vue. Ce manque vient de la complexion de notre organisme, les limitations de notre savoir dépendant de notre finitude.

Comme il est profond, ce mystère de l'Invisible ! Nous ne le pouvons sonder avec nos sens misérables, avec nos yeux qui ne savent apercevoir ni le trop petit, ni le trop grand, ni le trop près, ni le trop loin, ni les habitants d'une étoile, ni les habitants d'une goutte d'eau... avec nos oreilles qui nous trompent, car elles nous transmettent les vibrations de l'air en notes sonores. Elles sont des fées qui font ce miracle de changer en bruit ce mouvement et par cette métamorphose donnent naissance à la musique, qui rend chantante l'agitation muette de la nature... avec notre odorat, plus faible que celui du chien, avec notre goût, qui peut à peine discerner l'âge d'un vin! (II, 914)

Dans cette description du fonctionnement des sens, on peut remarquer un ordre de préséance : on commence par l'œil. Lorsque le narrateur dépeint une nouvelle vanité de l'homme imparfait, il s'en prend encore à l'œil.

Et je songeais encore : mon œil est si faible, si imparfait, qu'il ne distingue même point les corps durs s'ils sont transparents comme le verre !... Qu'une glace sans tain barre mon chemin, il me jette dessus comme l'oiseau entré dans une chambre se casse la tête aux vitres. Quoi d'étonnant, alors, à ce qu'il ne sache pas apercevoir un corps nouveau que la lumière traverse. (II, 934)

9 Ces deux passages font écho à un discours tenu par le « malade » du « Horla » de 1886 :

Mais notre œil, messieurs, est un organe tellement élémentaire qu'il peut distinguer à peine ce qui est indispensable à notre existence. Ce qui est trop petit lui échappe, ce qui est trop grand lui échappe, ce qui est trop loin lui échappe. Il ignore les milliards de petites bêtes qui vivent dans une goutte d'eau. Il ignore les habitants, les plantes et le sol des étoiles voisines ; il ne voit pas même le transparent. Placez devant lui une glace sans tain parfaite, il ne la distinguera pas et nous jettera dessus comme l'oiseau pris dans une maison, qui se casse la tête aux vitres. (II, 829)

10 Je propose de comparer l'œil des Horla avec celui de « Un Cas de divorce ». On y lit :

Oh ! mystère ! Quel mystère ? L'œil ?... Tout l'univers est en lui, puisqu'il le voit, puisqu'il le reflète. Il contient l'univers, les choses et les êtres, les forêts et les océans, les hommes et les bêtes, les couchers de soleil, les étoiles, les arts, tout, tout, il voit, cueille et emporte tout ; et il y a plus encore en lui, il y a l'âme, il y a l'homme qui pense, l'homme qui aime, l'homme qui rit, l'homme qui souffre ! oh ! 
regardez les yeux bleus des femmes, ceux qui sont profonds comme la mer, changeants comme le ciel, si doux, si doux, doux comme les brises, doux comme la musique, doux comme les baisers, et transparents, si clairs qu'on voit derrière, on voit l'âme, l'âme bleue qui les colore, qui les anime, qui les divinise. Oui, l'âme a la couleur du regard. L'âme bleue seule porte en elle du rêve, elle a pris son azur aux flots et à l'espace. L'œil ! Songez à lui ! L'œil ! Il boit la vie apparente pour en nourrir la pensée. Il boit le monde, la couleur, le mouvement, les livres, les tableaux, tout ce qui est beau et tout ce qui est laid, et il en fait des idées. (II, 779) constatons que la vision subsume l'ensemble des autres sens. Les références à l'œil viennent toujours en première place ${ }^{4}$; les regards « doux comme la musique, doux comme des baisers » contiennent les actions de l'ouïe et du toucher. Enfin, la vue amène le paradigme de l'actio in distans qui structure la faculté d'abstraction : « Il boit le monde [...] et il en fait des idées ", celles-ci sont tirées de l'expérience ${ }^{5}$. La pensée du réel s'enracine dans sa perception sensitive, dont le modèle est la vue.

Autrement dit, "l'univers ", le monde sont dans la dépendance du regard. Le visible stimule l'âme ; il devient le réel, et, uniment, le rationnel. Du coup, l'invisibleinconnaissable ne peut passer pour du réel qu'à condition de supposer que cet invisible puisse être vu.

Vu par un œil d'œil une sorte d'œil idéal, meilleur en tout point à celui de l'homme ; changer d'œil revient à changer de (perception du) monde.

Donc cet organe [l'œil] impose à l'esprit sa manière de voir, ou mieux sa façon arbitraire de constater les dimensions et d'apprécier les rapports de la lumière et de la matière. (II, 463 ; « Lettre d'un fou »)

$\mathrm{Vu}$ par l'instrument, aidé de la réflexion scientifique. Avant la séance d'hypnose du Horla, le $\mathrm{D}^{\mathrm{r}}$ Parent prétend que, confronté « au mystère impénétrable pour ses sens grossiers et imparfaits » (II, 922), l'homme " tâche de suppléer, par l'effort de son intelligence, à l'impuissance de ses organes " (ibid.). Le positivisme du praticien laisse entendre que l'invisible et l'inconnaissable ne le sont que temporairement. Dans l'avenir, on comprendra davantage, on pressentira, on sentira presque, on sera tout proche de voir le mystérieux. Dans une chronique intitulée "Au Muséum d'histoire naturelle ", Maupassant écrit :

On marche dans le certain, vers le certain; on sait que tout effet a une cause logique, et que, si cette cause nous échappe, c'est uniquement parce que notre esprit, notre pénétration, nos organes et nos instruments sont trop faibles. $(1,187)$

Dans toute l'œuvre de Maupassant se lit le sentiment d'assister à un moment de transition entre les peurs irrationnelles et la science future. Ainsi, la maladie inintelligible est en train de se transformer en un microbe guérissable. « La Peur » exprime cette ambivalence :

Le choléra, c'est autre chose, c'est l'Invisible, c'est un fléau d'autrefois, des temps passés, une sorte d'Esprit malfaisant qui revient et qui nous étonne autant qu'il nous épouvante, car il appartient, semble-t-il, aux âges disparus. (II, 204), C'est qu'Il est là, qu'on le brave, non pas le Microbe, mais le Choléra, et qu'on veut être crâne devant lui, comme auprès d'un ennemi caché qui vous guette. C'est pour lui qu'on danse, qu'on rit, qu'on crie, qu'on allume ces feux, qu'on joue ces valses, pour lui, l'Esprit qui tue, et qu'on sent partout présent, invisible, menaçant, comme un de ces anciens génies du mal que conjuraient les prêtres barbares... (II, 205)

Là où, par réflexe, les hommes expriment leur angoisse du Choléra, les savants combattent le Microbe. Celui-ci sort désormais du domaine de l'invisible, il est avéré par 
le microscope. Ce qui n'était pas visible à l'œil nu (et qui, partant, restait douteux) acquiert une existence irréfutable à partir du moment où il s'annule structurellement. Je veux dire : on est sûr qu'il y a de l'invisible dès lors qu'il se révèle visible.

Cet effacement interne sape dorénavant la croyance.

Lentement, depuis vingt ans, le surnaturel est sorti de nos âmes. (2, 256 ; Le Fantastique)

C'est fini, fini. Les choses ne parlent plus, ne chantent plus, elles ont des lois ! (1, 314)

La seconde citation est extraite d'« Adieu mystères » (1881), où se développe un discours confiant et rationaliste. Dans " Le Fantastique » $(2,256260)$, chronique légèrement postérieure, Maupassant semble même prophétiser la fin du fantastique. Mais il annonce surtout la mort d'un genre littéraire fondé sur une crédulité ancestrale et qui ne se sépare pas de son cortège de monstres prêts à l'emploi, de ses trucs de conteur, de ses règles et lois. Maupassant fait signe vers un nouveau fantastique, étayé par l'effroi du réel. Face à " tous ces voiles levés » $(1,314)$ par la science, y a-t-il pour autant une complète apocalypse du mystérieux? Nouveau credo.

Il me semble qu'on a dépeuplé le monde. On a supprimé l'Invisible. Et tout me paraît muet, vide, abandonné ! Quand je sors la nuit, comme je voudrais pouvoir frissonner de cette angoisse qui fait se signer les vieilles femmes le long des murs des cimetières, et se sauver les derniers superstitieux devant les vapeurs étranges des marais et les fantasques feux follets. Comme je voudrais croire à ce quelque chose de vague et de terrifiant qu'on s'imaginait sentir passer dans l'ombre ! Comme les ténèbres des soirs devaient être plus noires autrefois, grouillantes de tous ces êtres fabuleux! $(1,314$ et suiv.)

Le progrès scientifique est un dépeupleur et l'homme nouveau arrive dans un monde déserté. Or, d'aphorisme à aphorisme, le narrateur du Horla donne l'impression de répondre à la chronique :

Quand nous sommes seuls longtemps, nous peuplons le vide de fantômes. (II, 921)

Ce vide, acquis à force de violence contre l'invisible, aurait besoin d'être (re)peuplé. Par des fantômes, qu'est-ce à dire ? Le narrateur poursuit son hypothèse psychologique :

On dirait que l'homme, depuis qu'il pense, a pressenti et redouté un être nouveau, plus fort que lui, son successeur en ce monde, et que le sentant proche et ne pouvant prévoir [ne pouvant pré-voir] la nature de ce maitre, il a créé, dans sa terreur, tout le peuple fantastique des êtres occultes, fantômes vagues nés de la peur. (II, 931)

21 Il y eut ainsi un premier peuple d'êtres fantastiques : pure illusion. Puis il y eut la rationalisation qui fit disparaître ces chimères. Avant qu'arrive l'Être Nouveau, le Horla. Cet Invisible, qui vient après l'invisible rendu visible, et qui restera tel, nous regarde tous : le cocher du narrateur " souffre du même mal » en l'absence de son maître (II, 918 et suiv.), les Brésiliens sont condamnés (II, 932) : le mal se propage. Cet Invisible nous regarde, tapi dans l'ombre.

Il ne se manifeste plus, mais je le sens près de moi, m'épiant, me regardant, me pénétrant, me dominant et plus redoutable, en se cachant ainsi, que s'il signalait par des phénomènes surnaturels sa présence invisible et constante. (II, 929)

Pour voir pleinement, il convient d'être invisible. Le « Oh ! mystère ! Quel mystère ? (II, 779) que nous avions laissé tout à l'heure serait là pour nous indiquer que le mystère, donc l'inconnaissable, donc l'invisible, est la condition de l'usage de l'œil. En ce cas, cet 
organe peut effectivement voir et « [boire] la vie apparente » (ibid.) du narrateur du Horla. L'œil invisible permet le contrôle sur l'être épié. épisode, qui fut qualifié par André Targe de " sommet et catalyse ${ }^{6}$ » du récit, a été étudié de nombreuses fois, en particulier d'un point de vue structural. Dans les contraintes qui sont les nôtres, on commencera par simplement reproduire le « noyau de cette scène ».

Je me dressai, les mains tendues, en me tournant si vite que je faillis tomber. Eh bien ?... on y voyait comme en plein jour, et je ne me vis pas dans ma glace! Elle était vide, claire, profonde', pleine de lumière! Mon image n'était pas dedans... et j'étais en face, moi! Je voyais le grand verre limpide du haut en bas. Et je regardais cela avec des yeux affolés ; et je n'osais plus avancer, je n'osais plus faire un mouvement, sentant bien pourtant qu'il était là, mais qu'il m'échapperait encore, lui dont le corps imperceptible avait dévoré mon reflet.

Comme j'eus peur ! Puis voilà que tout à coup je commençai à m'apercevoir dans une brume, au fond du miroir, dans une brume comme à travers une nappe d'eau ; et il me semblait que cette eau glissait de gauche à droite, lentement, rendant plus précise mon image, de seconde en seconde. C'était comme la fin d'une éclipse. Ce qui me cachait ne paraissait point posséder de contours nettement arrêtés, mais une sorte de transparence opaque, s'éclaircissant peu à peu.

Je pus enfin me distinguer complètement, ainsi que je le fais chaque jour en me regardant.

Je l'avais vu ! L'épouvante m'en est restée, qui me fait encore frissonner. (II, 935 et suiv.)

Dans « Le Horla », la première version de 1886, Maupassant écrivait « l'apparition » dans des termes très proches (cf. II, 828). Encore précédemment, le thème se trouvait dans « La Lettre d'un fou ».

Je me dressai en me tournant si vite que je faillis tomber. On y voyait comme en plein jour, et je ne me vis pas dans la glace! Elle était vide, claire, pleine de lumière. Je n'étais pas dedans, et j'étais en face, cependant. Je la regardais avec des yeux affolés. Je n'osais pas aller vers elle, sentant bien qu'il était entre nous, lui, l'Invisible, et qu'il me cachait.

Oh ! comme j'eus peur ! Et voilà que je commençai à m'apercevoir dans une brume au fond du miroir, dans une brume comme à travers de l'eau ; et il me semblait que cette eau glissait de gauche à droite, lentement, me rendant plus précis de seconde en seconde. C'était comme la fin d'une éclipse. Ce qui me cachait n'avait pas de contours, mais une sorte de transparence opaque s'éclaircissant peu à peu.

Et je pus enfin me distinguer nettement, ainsi que je le fais tous les jours en me regardant.

Je l'avais donc vu !

Et je ne l'ai pas revu. (II, 466)

En dépit de ces deux dernières phrases, le fou épistolier notait un peu plus haut : «Et j'ai vu - j'ai vu un être invisible - autant qu'on peut les voir, ces êtres. » (II, 465). Une 
restriction s'impose bel et bien, car, dans les trois cas, l'Être apparaît plus qu'on ne le voit. Même s'il tombe dans un piège qui lui est tendu, le Horla n'est pas simplement vu. Le ralentissement de la narration - obtenu par la désignation de chaque détail -, certaines formules (" de seconde en seconde ", " peu à peu », « enfin ») indiquent que l'épiphanie est un processus non instantané. Donc, en tout état de cause, le Je ne voit pas immédiatement le Horla (cf. le rôle du miroir).

Ensuite, et je vais développer ce point, les yeux du narrateur sont curieusement perturbés. Ils voient ; plus précisément, ils ne voient pas l'image ; on en conclut qu'ils voient. Ils voient sans voir ; ils ne voient rien mais voient. Puisque nous ne sommes alors ni dans le voir, ni dans le non-voir, je propose d'appeler cette configuration le voir non voir.

Dès lors, par rétrospection, nous retrouvons ce voir non voir dans tout Le Horla. Chaque fois, il est associé à l'Être Redoutable. La première manifestation extraordinaire est relatée le cinq juillet. Après un sommeil épouvantable, le narrateur se réveille et décide de boire de l'eau :

[...] j'allumai une bougie et j'allai vers la table où était posée ma carafe. Je la soulevai en la penchant sur mon verre ; rien ne coula. - Elle était vide ! Elle était vide complètement ! [...] On avait donc bu cette eau ? Qui ? Moi ? moi, sans doute ? (II, 919)

Cette absence de liquide dans une carafe qu'on avait vue pleine avant le coucher est au début d'une série d'expériences menées jusqu'au dix juillet (voir II, 920). "Quelqu'un » boit le lait et l'eau du narrateur ; ce qui ne manque pas d'effrayer celui-ci. À l'issue de sa première découverte, il consigne :

Ah! qui comprendra mon angoisse abominable? Qui comprendra l'angoisse d'un homme, sain d'esprit, bien éveillé, plein de raison et qui regarde épouvanté, à travers le verre d'une carafe, un peu d'eau disparue pendant qu'il a dormi! (II, 920)

31 À ma connaissance, ce passage n'a jamais surpris outre mesure les commentateurs. Pourtant, relisons :

qui regarde [...] un peu d'eau disparue.

32 Je ne regarde pas l'eau qui reste, je ne regarde pas la carafe à moitié vide, je regarde l'eau disparue, je regarde ce qui n'est plus là, je ne regarde rien. Insistons sur l'impossibilité logique : j'ai la capacité de voir quelque chose lorsque je ne le vois pas. Les actions du Horla, on ne peut que les voir non voir. Il est vrai que depuis qu'il est « souffrant » (II, 914), qu'il est « malade, décidément » (ibid.), le narrateur a « l'œil dilaté » (II, 915). Cette fièvre étrange, cet étranger dans mon corps changent ma vision, je suis écarquillé, avec « tous mes organes surexcités $\left(\right.$ (II, 935) ${ }^{8}$.

Une même hyperesthésie explique les prouesses de $\mathrm{M}^{\mathrm{me}}$ Sablé, dès qu'elle se trouve sous hypnose.

Je voyais les yeux de $\mathrm{M}^{\text {me }}$ Sablé s'alourdir, sa bouche se crisper, sa poitrine haleter.

Au bout de dix minutes, elle dormait. [Elle a donc vraiment les yeux clos.]

« Mettez-vous derrière elle », dit le médecin.

Et je m'assis derrière elle. Il lui plaça entre les mains une carte de visite en

lui disant : «Ceci est un miroir ; que voyez-vous dedans?»

Elle répondit :

« Je vois mon cousin ». [C'est-à-dire le narrateur.]

- «Que fait-il?

- Il se tord la moustache.

- Et maintenant?

- Il tire de sa poche une photographie. 


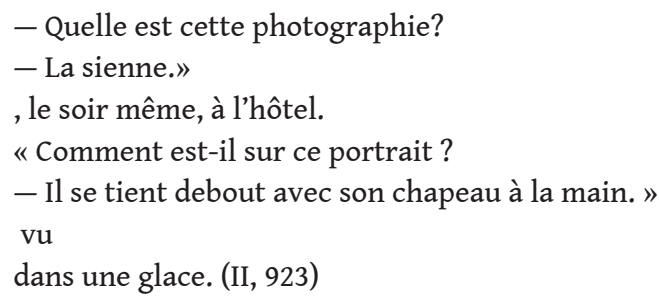
propice à l'effroi, et la calme météorologique à la sérénité. Ici, rien de définitif. Le Mont venté est l'occasion d'un répit, et l'absence de vent doit inquiéter : elle risque toujours de trahir la présence du Horla. Attendant que l'incendie détruise sa maison et son ennemi, le narrateur trouve que " tout était noir, muet, immobile » (II, 937). Il ajoute : " pas un souffle d'air ». Et justement, il est probable que le Horla vive encore (II, 938) ; et justement, il y avait « des montagnes de nuages qu'on ne voyait point, mais qui pesaient sur mon âme si lourds, si lourds » (II, 937). Des nuages qu'on ne voit pas mais qu'on voit quand même.

Comme un grand miroir, Le Horla fait réflexion pour la définition du fantastique, qui est le lieu utopique du voir non voir. Le fantastique, dont la hantise par l'Être redoutable forme l'expression, jaillit de la constriction de l'apparaître. Il soutient la phénoménologie (volontiers négative) de la vision, qui ne se limite pas à l'anatomie de l'œil. Par là, le fantastique est naturellement en émergence ; jamais stable, il est sur le point de se révéler 
et son exposition conditionne l'éclipse. Le repérage du fantastique viserait à le tuer, tout discours critique risquant de préférer le voyant à l'occulte. Mais il faut croire que l'attention portée à ce phénomène de visibilité est un des rares remèdes. Et que par le retour à l'origine non originaire de la vision, on prépare une appréhension possible du fantastique qui fait pièce à la norme et place à la continuité du réel.

\section{BIBLIOGRAPHIE}

ARISTOTE, De l'Âme [Traduction E. Barbotin ; texte établi par A. Jannone], Paris, Les Belles Lettres, « Collection des Universités de France », 1966.

MAUPASSANT Guy de, Contes et Nouvelles [édition de Louis Forestier], Paris, Gallimard, « Bibliothèque de la Pléiade ", 1974-1979, 2 vol.

Maupassant Guy de, Chroniques, Paris, UGE, 10/18, « Fins de siècles », 1980, 3 vol.

MAUPASSANT Guy de, Le Horla [édition d'Y. Leclerc], Paris/Cardeilhan, CNRS Zulma, « Manuscrits ", 1993.

TARGE André, « Trois apparitions du Horla », Poétique, n 74, 1975, p. 446-459.

\section{NOTES}

1.De façon générale, Le Horla désigne le texte de 1887, et « Le Horla » la première version (1886).

2.Je renvoie, pour ce qui est des contes et des nouvelles, à l'édition de la Pléiade. Je désigne la tomaison en chiffres romains, suivie du numéro de page. La référence aux Chroniques (en 10/18) se conforme au modèle suivant : tomaison en chiffres arabes, numéro de page.

3.Le soulignement à l'intérieur des citations est toujours de mon fait; en revanche, les caractères italiques et gras sont reproduits d'après le texte de l'édition utilisée.

4.Rien de plus traditionnel que cet ordre, déjà adopté par Aristote. Cf., par exemple, De l'Âme, II, 5-12 (416b-424b), où la faculté sensitive comporte d'abord la vue (II, 7).

5.Cette présentation n'est pas sans rappeler, évidemment, la théorie empiriste des idées.

6. « Trois apparitions du Horla », p. 449.

7.Je rétablis la virgule (entre « claire » et « profonde ») omise dans la Pléiade; cf. p. 32 du manuscrit de Maupassant, dans l'édition du Horla chez Zulma.

8.De même, la narrateur de «Lettre d'un fou » voit l'invisible à force « d'aiguiser [ses] organes, de les exciter » (II, 464). Et il note, avant la disparition du reflet : «J'avais des yeux étranges et les pupilles très dilatées. » (II, 465).

9.J'analyse ces relations dans ma thèse, encore en cours, et qui porte sur la possession et la lecture chez Maupassant, Artaud et Blanchot. 


\section{RÉSUMÉS}

Dans son étymologie et son émergence, le fantastique a trait à la question de la vision, qu'il thématise et interroge. De manière exemplaire, le fantastique chez Maupassant se concentre sur l'invisible et son mystère. En passant par la transformation que les révélations de la science (positive et médicale) font subir à la construction du monde, l'écrivain fait signe vers un nouveau fantastique. Un fantastique débarrassé des anciennes croyances en l'autre monde et qui repose sur un mode inédit d'invisibilité. Ainsi, Le Horla, qui peut apparaître comme une tentative de repeuplement du monde que la science a rationalisé, ouvre la voie à une catégorie nouvelle : le « voir non voir ».

Maupassant and the fantastic vision. - In its etymology and in its emergence, the fantastic genre deals with the issue of vision, which it brings to the status of theme and interrogates. In an exemplary fashion, the fantastic in the works by the French writer Guy de Maupassant concentrates itself on the invisible and its mystery. By passing through the transformation that the revelations of science - both practice and medical science - exercised on the world construction, this author produces a sign directed towards a new fantastic genre. That is, a fantastic genre liberated from old beliefs which is based on a new invisibility mode. Thus, the Horla (Maupassantís famous fantastic short story), which may be considered as an attempt to repopulate the world that science has rationalised, paves the way for a new category : the "voir non voir" (literally " to see not to see ").

\section{AUTEUR}

\section{LAURENT DUBREUIL}

Laurent DubreuIL a vingt-cinq ans. Ancien élève de l'École normale supérieure, il est agrégé de lettres. Ses recherches portent principalement sur les expériences d'écriture philosophique et littéraire. Sa thèse de doctorat traite des rapports entre la lecture et la possession (chez Maupassant, Artaud et Blanchot). En parallèle, il étudie, sous la direction d'Hélène Cixous, la construction de la notion d'amitié dans le corpus grec et sa postérité. 